\title{
Histoire des facultés de théologie des universités publiques en France
}

Brigitte Basdevant-Gaudemet

\section{(2) OpenEdition \\ 12 Journals}

Édition électronique

URL : https://journals.openedition.org/rdr/1429

DOI : $10.4000 /$ rdr.1429

ISSN : 2534-7462

Éditeur

Presses universitaires de Strasbourg

\section{Édition imprimée}

Date de publication : 6 mai 2021

Pagination : 23-40

ISBN : 979-10-344-0089-8

ISSN : 2493-8637

Référence électronique

Brigitte Basdevant-Gaudemet, «Histoire des facultés de théologie des universités publiques en France », Revue du droit des religions [En ligne], 11 | 2021, mis en ligne le 06 mai 2021, consulté le 04 mai 2022. URL : http://journals.openedition.org/rdr/1429; DOI : https://doi.org/10.4000/rdr.1429

\section{(c) $(1)(9$}

La revue du droit des religions est mise à disposition selon les termes de la Creative Commons Attribution - Pas d'Utilisation Commerciale 4.0 International - CC BY-NC 4.0. 


\section{Histoire des facultés de théologie des universités publiques en France}

\section{Brigitte BASDEVANT-GAUDEMET}

Université Paris-Saclay, Droit et Sociétés religieuses (DSR)

\section{RÉSUMÉ}

L'histoire propre à la France explique que, contrairement aux autres pays européens, l'Université ne compte aucune faculté de théologie, sauf à Strasbourg. Au sein de l'Université napoléonienne, plusieurs facultés de théologie catholique et protestante furent érigées et le culte juif eut son organisation. Ces établissements fonctionnèrent souvent mal, surtout les facultés catholiques pour deux raisons: méfiance de la part des évêques et aucune reconnaissance par Rome malgré les tentatives de négociations. En 1875, la création d'universités privées ne fit qu'accroître la confusion, jusqu'à la suppression du budget des facultés de théologie de l'Université de France en 1885, entraînant leur disparition immédiate. À Strasbourg, la faculté de théologie protestante est rétablie dans l'Université dès 1872; celle de théologie catholique est érigée en 1902, en accord avec Rome.

\section{Abstract}

France's history explains that, unlike other European countries, there is no faculty of theology at the public university, except in Strasbourg. Within the Napoleonic University, several Catholic and Protestant theological faculties were erected and the Jewish faith had its own organization. These institutions often ran poorly, especially the Catholic faculties, for two reasons: mistrust on the part of the bishops and no recognition by Rome despite attempts to negotiate. In 1875, the creation of private universities only increased the confusion, until the removal of funding for the public faculties of theology in 1885, resulting in their immediate disappearance. In Strasbourg, however, the faculty of Protestant theology was restored at the University in 1872; the faculty of Catholic theology was erected in 1902 in agreement with Rome. 
T 'existence de facultés de théologie au sein des universités publiques est, Lde nos jours, exceptionnelle en France et les études ici réunies éclaireront cette réalité. Notre propos ne consiste pas à analyser la situation actuelle, mais à donner un bref éclairage historique. Nous n'évoquerons pas la splendeur des universités médiévales regroupant généralement les facultés de théologie, de droit, des arts et de médecine. On n'admirera jamais assez l'essor de ces prestigieuses universités à Bologne ${ }^{1}$, Cracovie, Budapest, Oxford, Paris $^{2}$ ou Montpellier et rapidement à travers tout l'Occident. Les universités étaient aux mains des clercs. Les maîtres étaient tous membres du clergé et plusieurs universités n'acceptaient comme écoliers que des candidats également engagés dans la cléricature. L'autorité supérieure revenait aussi à un haut dignitaire ecclésiastique, souvent l'évêque, parfois directement le pontife romain. Rois, princes ou pouvoirs municipaux entendaient avoir, eux aussi, d'importantes prérogatives sur ce haut lieu du savoir et sur la population estudiantine, parfois tumultueuse, qui l'animait. Les statuts de chaque université étaient fixés par des chartes de franchises âprement négociées entre tous les intéressés et que maîtres et écoliers invoquaient afin de protéger leurs libertés. L'apogée médiéval fut suivi par un certain déclin, dès le XIV siècle et peut-être davantage par la suite. La faculté de théologie de Paris, entre autres, fut le théâtre d'oppositions d'écoles peu favorables à l'essor scientifique. Les doctrines se déchiraient entre la via antiqua s'appuyant sur Thomas d'Aquin et la via moderna, proche du nominalisme anglo-saxon. Pendant les guerres de religion, l'Université avait pris parti pour la Ligue et l'enseignement en souffrit considérablement. Elle fut discréditée, parfois méprisée. L'université de Paris reçut de nouveaux statuts en 1598, accordés unilatéralement par Henri IV, en dehors de toute consultation du pape. Par la suite, nombreux furent ceux affirmant que les idées nouvelles naissent en dehors de ses murs.

En 1642, traitant de la souveraineté royale et faisant fi des réalités de l'histoire, Cardin Le Bret ne craint pas d'affirmer: "De toute antiquité il n'appartient qu'aux princes souverains d'établir des Universités. ${ }^{3}$ »Pour ce théoricien de la puissance publique, les universités sont des corps et il relève de la compétence exclusive du souverain d'autoriser la création de nouveaux corps dans son royaume. Un siècle plus tard, Malesherbes ajoutera qu'il ne suffit pas que l'Université soit publique, mais qu'elle doit, en outre, être

1. Studi e memorie per la storia dell'Università di Bologna, 1909.

2. J. Gaudemet, «Les débuts de l'Université de Paris» et «La vie universitaire à Paris (XII ${ }^{\mathrm{e}}$ XIII ${ }^{\mathrm{e}}$ s.) », in Église et société en Occident au Moyen Âge, London, Variorum Reprints, 1984, ch. VIII et IX.

3. C. Le Bret, De la souveraineté du roi, Paris, 1642, liv. IV, chap. XIV. 
dirigée par l'autorité souveraine. Aussi gallican que Le Bret ou Malesherbes, le Parlement de Paris, dans un arrêt de 1667, déclare nulle une nomination d'un maître en théologie, obtenue en cour de Rome et contestée par son rival qui soutient que l'Université est d'institution royale et de patronage laique non ecclésiastique. Les plaidoyers sont intéressants et portent sur ce caractère, ecclésiastique ou non, de l'Université. Toutefois, le Parlement se garde bien de trancher expressément la question de savoir «si l'Université doit être estimée et réputée ecclésiastique ou laïque ou un corps mixte ${ }^{4}$, mais il donne raison au candidat du camp laique. Précisons que si le terme laïque est constamment utilisé par les juristes dans cette affaire, il ne faut certainement pas entendre cette laïcité comme excluant le caractère religieux, et plus précisément catholique, de l'institution, mais comme signifiant que les autorités ayant pouvoir sur l'Université sont le roi et ses agents, à l'exclusion des membres du clergé ou du pape ${ }^{5}$.

Dans le domaine de l'enseignement, la crise révolutionnaire fut plus propice aux projets qu'aux réalisations concrètes et durables. Un décret du 17 octobre 1790 ferma les écoles de la Sorbonne, ce qui mit fin à tout enseignement de la théologie à Paris, mesure complétée par le décret du 15 septembre 1793 supprimant les facultés de théologie, de droit, de médecine et des arts dans toute la République. Des écoles furent néanmoins créées, importantes et parfois durables et le mouvement se poursuivit sous le Directoire, mais ces établissements ne s'occupaient pas de théologie. Au lendemain de ces dix années tumultueuses, la question déjà présente sous l'Ancien Régime se posa à nouveau, mais sous un autre jour: l'enseignement, notamment l'Université, doit-il dépendre de l'État ou de l'Église? Si à l'époque médiévale l'Église avait pu régner en maître sur l'éducation, ce monopole avait progressivement été quelque peu atteint par la monarchie, mais une monarchie de droit divin, aux mains d'un Roi Très Chrétien agissant dans le cadre d'une politique gallicane. À cette collaboration entre les deux puissances, certes pas toujours harmonieuse et pourtant généralement acceptable, les révolutionnaires ont substitué un esprit de rivalités et d'oppositions.

Tel est le contexte dans lequel Napoléon va organiser l'Université impériale: une tradition multiséculaire d'un enseignement aux mains de l'Église, brutalement mise à mal par un gouvernement révolutionnaire soucieux de

4. Arrêt du Parlement de Paris du $1^{\text {er }}$ avril 1667 : Journal des Audiences, t. II, 1733, p. 549-550.

5. Pour un aperçu rapide de l'histoire de ces établissements en Europe: B. BASDEVANT-GAUDEMET, «Histoire du droit ecclésiastique en Europe, une discipline universitaire», in J.-M. Gonzàlez del Valle et a. Hollerbach (dir.), L'enseignement du droit ecclésiastique de l'État dans les universités européennes, Leuven, Peeters, 2005, p. 1-54. 
former le citoyen. Le changement est particulièrement important en ce qui concerne l'enseignement de la théologie. L'histoire spécifique à la France, celle des dix années révolutionnaires, mais plus encore celle de l'ensemble du XIX ${ }^{e}$ siècle où gouvernements cléricaux et anticléricaux se succèdent et s'affrontent, va avoir de fortes incidences sur les facultés de théologie. Dans la quasi-totalité des pays européens au XIX $x^{e}$ siècle comme encore de nos jours, les universités publiques accueillent en leur sein des facultés de théologie; en outre, le plus souvent, les facultés de théologie catholique insérées dans une université publique bénéficient également d'une reconnaissance de la part du Saint-Siège. L'histoire propre à la France a placé notre pays dans une autre situation. Les changements dans l'organisation de l'enseignement au XIX ${ }^{e}$ siècle ont été particulièrement tributaires des constants retournements politiques du pays. Reprenons cette histoire, selon son déroulement chronologique. Au cours des deux siècles écoulés depuis la création de l'Université napoléonienne (1), les débuts de la Troisième République marquent un tournant notable (2).

\section{LES FACULTÉS DE THÉOLOGIE DANS LE CADRE DE L'UNIVERSITÉ IMPÉRIALE : INSTAURATION ET FONCTIONNEMENT JUSQU'EN 1870}

\subsection{L'INSTAURATION DES FACULTÉS DE L'UNIVERSITÉ PUBLIQUE}

Avant même la création de l'Université napoléonienne, la loi du 18 germinal an X (8 avril 1802), regroupant le concordat de juillet 1801, les articles organiques du culte catholique et les articles organiques des cultes protestants, avait posé les bases du système futur. À cette date, le culte juif n'était pas pris en considération. Protestants et catholiques voyaient se dessiner les établissements qui allaient prendre vie.

Les articles organiques des cultes protestants, dans leurs dispositions générales relatives à toutes les communions protestantes confortaient ou créaient des établissements qui, de fait, fonctionneront. «Il y aura deux académies ou séminaires dans l'est de la France, pour l'instruction des ministres de la Confession d'Augsbourg» (art. 9). «Il y aura un séminaire à Genève pour l'instruction des ministres des églises réformées» (art. 10). Les autres dispositions précisaient que la nomination des professeurs relevait du Premier consul et que nul ne pourrait être ministre ou pasteur de ces Églises sans avoir étudié dans ces académies ou séminaires. Était également précisée la nécessité de soumettre à approbation du Gouvernement tout règlement intérieur relatif à l'organisation de l'enseignement dispensé, à son contenu, à la 
qualité des professeurs et aux «attestations d'étude, de bonne conduite et de capacité » (art. 14). Le ton était donné et le détenteur de l'autorité clairement identifié. Les villes choisies étaient Strasbourg et Genève ${ }^{6}$, décision répondant à une certaine logique, car ces enseignements y avaient connu un passé remarquable. La séance inaugurale de l'académie luthérienne de Strasbourg se déroula le 19 septembre 1803 et les cours commencèrent en novembre de la même année ${ }^{7}$. L'académie prend le nom de séminaire protestant en 1808. Jusqu'en 1905, l'exigence de ces titres universitaires demeura pour qui voulait exercer un ministère pastoral, luthérien ou réformé. Le diplôme de bachelier en théologie était indispensable pour obtenir confirmation de ses fonctions par le Gouvernement.

La formation des ministres du culte catholique n'était envisagée que de façon assez imprécise par la loi de germinal, flou qui reflétait les préoccupations de chacun: du côté de l'Église, les évêques souhaitaient des prêtres qui soient des pasteurs zélés et attentifs, chez qui la soumission devait être une qualité qui l'emporterait sur l'instruction. Les pouvoirs publics se souciaient eux aussi d'une obéissance, due sans doute à l'Église, mais plus encore à l'État. Dans cet esprit, concordat et articles organiques toléraient l'existence de séminaires diocésains ${ }^{8}$, à condition que la Déclaration des quatre articles y soit enseignée, ce dont les évêques devaient rendre compte au conseiller chargé des cultes ${ }^{9}$. Au cours du XIX ${ }^{e}$ siècle, l'enseignement de cette charte du gallicanisme telle qu'elle avait été élaborée par l'Assemblée du clergé de France en 1682 sous Louis XIV fut peu assuré, mais la prescription fut souvent invoquée, par les uns ou les autres, pomme de discorde pouvant alimenter tous les débats.

Si les séminaires diocésains ne rencontrèrent guère de faveurs, au lendemain de la publication de la loi Bonaparte demanda à Jean Étienne Marie Portalis, conseiller d'État chargé des cultes et principal auteur des articles organiques, de prévoir des établissements dispensant un enseignement de la théologie catholique plus affiné que celui assuré au sein des séminaires diocésains; l'État s'engagerait à les financer ce qui lui en assurerait parallèlement le contrôle. Après consultation de hauts dignitaires ecclésiastiques,

6. Genève avait été réunie à la France en 1798 et l'enseignement de la religion luthérienne y jouissait déjà d'une grande réputation.

7. B. Neveu, Les facultés de théologie catholique de l'Université de France (1808-1885), Paris, Klincksieck, 1998.

8. Concordat, art. 11 : les évêques «pourront» avoir un séminaire, «sans que le gouvernement s'oblige à les doter»; repris aux articles organiques, art. 11.

9. Art. 24 des articles organiques. 
Portalis propose un projet dans lequel l'enseignement est placé «sous la surveillance du magistrat politique comme l'était celui des universités qu'il remplace», dit-il dans son rapport; toutefois, ajoute-t-il «il ne saurait être étranger à la sollicitude des évêques». État et Église collaboreront, dans un système où la prépondérance du Gouvernement est clairement affirmée et justifiée par le financement qu'accorderait l'État. Par la loi du 14 mars 1804 sont effectivement créés des séminaires ou maisons d'instruction dans chaque circonscription métropolitaine qui, comme les récentes écoles de jurisprudence et de médecine, ont vocation à s'insérer dans le cadre de l'Université future. Les directeurs et professeurs y seront nommés par le Premier consul, sur indications données par l'archevêque (art. 6).

Ces établissements, protestants et catholiques, durent prendre place dans le cadre de l'Université impériale ${ }^{10}$. Celle-ci vit tout naturellement le jour en harmonie avec les principes généraux de l'organisation administrative voulue par le Premier consul: centralisation, autorité, étatisation. Telles étaient déjà les idées directrices de la loi Fourcroy du $1^{\text {er }}$ mai 1802 instaurant les lycées. L'Université impériale est créée par la loi du 10 mai 1806. Elle est organisée en 1808, par plusieurs décrets confortant la tendance d'étatisation initiée par la loi Fourcroy. Fidèle, là encore, à sa méthode d'administration, Napoléon puise pour partie à l'Ancien Régime et pour partie à son sens de l'autorité. Il tente de combiner la tradition des corporations universitaires de l'ancienne société et la notion de service public étroitement contrôlé par l'État, cette seconde préoccupation l'emportant nettement sur la première. Il existera une seule Université impériale, chargée de dispenser les enseignements des divers niveaux et diverses matières à travers tout l'empire. Aux multiples universités médiévales, organisées en corporations jalouses, chacune, de leurs privilèges, l'empire substitue le monopole d'un service public de l'enseignement devant former le citoyen au service de l'État et du régime. Cette université aura le monopole de l'attribution des grades aux élèves et étudiants. Un enseignement privé subsiste, mais placé sous la tutelle de l'État et contrôlé par les inspecteurs généraux de l'instruction publique. Certes, ce monopole sera progressivement atteint et le législateur organisera les divers niveaux de l'enseignement privé ${ }^{11}$. Toutefois, le principe d'une université publique centralisée et possédant le monopole de la collation des grades demeurera fondamental.

10. Étude générale dans F. Messner, P.-H. Prélot et J.-M. Woehrling (dir.), Traité de droit français des religions, Paris, LexisNexis, $2^{e}$ éd. 2013, no 3262 et $\mathrm{s}$.

11. Loi du 28 juin 1833, dite loi Guizot sur la liberté de l'enseignent primaire; loi du 15 mars 1850, dite loi Falloux sur la liberté de l'enseignement secondaire et loi du 12 juillet 1975, dite loi Dupanloup sur la liberté de l'enseignement supérieur. 
La loi du 10 mai 1806 créant l'université est plus que laconique et l'organisation dépend davantage du décret du 17 mars 1808 qui établit cinq facultés au sein de l'université. Les facultés de théologie catholique et protestante figurent au premier rang et sont accompagnées du droit, de la médecine, des sciences et des lettres. Le cadre ainsi posé ne connaîtra que des modifications mineures au cours du XIx ${ }^{e}$ siècle et même jusqu'au milieu du Xxe .

L'érection du service public de l'Université ne bouleverse pas totalement l'organisation de l'enseignement de la théologie protestante ${ }^{12}$. Le décret du 17 mars 1808 prévoit officiellement la faculté de théologie luthérienne au sein de l'université de Strasbourg et celle de théologie réformée à Genève. La faculté de Genève sera transférée à Montauban en 1815, après les modifications des frontières territoriales de la France. Elle fonctionnera plus d'un siècle à Montauban, jusqu'à son nouveau transfert à Montpellier en 1919; à Montauban, après quelques décennies peu brillantes, elle avait acquis, à la fin du $\mathrm{XIX}^{\mathrm{e}}$ siècle, une autorité scientifique réelle ${ }^{13}$. Il fallut quelques années pour que s'établisse à Strasbourg un équilibre entre luthériens et réformés. Le séminaire existant dépendait des luthériens. La faculté de théologie de l'université fut créée à côté de l'académie en $1818^{14}$, elle aussi essentiellement luthérienne, mais les réformés y auront une chaire de dogme dès 1820. Suite à la guerre contre la Prusse, cette faculté de théologie de Strasbourg sera recréée à Paris par décrets des 27 mars et $1^{\text {er }}$ octobre 1877. Pourtant, la faculté de théologie protestante parisienne connaîtra une modification substantielle: selon le vœu exprimé par le synode général de 1872, les chaires de cette faculté, désormais qualifiée de mixte, sont partagées en nombre égal entre les deux confessions protestantes, réformée et luthérienne, chacune ayant au moins une chaire de dogme.

La situation des séminaires catholiques métropolitains est de nouveau examinée lors de l'élaboration de la loi sur l'Université. En 1806, Napoléon aurait voulu qu'ils soient d'une part des séminaires dépendant de l'archevêque, mais aussi des écoles spéciales de théologie, pouvant conférer des grades attribués par une autorité étatique, comme les autres facultés de l'université; et Napoléon d'ajouter qu'il en allait ainsi dans l'ancienne France. Les prêtres seront formés par les évêques au séminaire, mais les

12. Rép. Dalloz, 1853, V० Culte, $\mathrm{n}^{\circ} 719$.

13. A. Gounelle, "La faculté de théologie protestante de Montauban», Études théologiques et religieuses, 2013-2, p. 233-255.

14. F. MESSNER, «Formation des ministres du culte et des cadres religieux en Alsace-Moselle», JCl. Alsace Moselle, 2017, fasc. 235, $\mathrm{n}^{\text {os }}$ 9-25. 
ecclésiastiques d'un degré supérieur devront posséder un grade, de bachelier, de licencié ou de docteur, octroyé dans le cadre de l'école spéciale de théologie agrégée à l'université. Les chaires de théologie ne seront confiées qu'à des clercs qui, en tant que professeurs d'université, ont la qualité d'agent du Gouvernement.

Des facultés de théologie catholique sont rapidement mises en place dans les académies d'Aix, Bordeaux, Lyon, Paris, Toulouse ${ }^{15}$ ainsi que Rouen, villes qui étaient toutes sièges d'archevêchés. Les candidats au professorat devaient être docteurs en théologie; suite à un concours évalué par les membres de la faculté, trois personnes seront présentées par l'évêque ou l'archevêque au grand-maître de l'université à qui revient le droit de nomination. Ce mode de désignation resta, théoriquement, en vigueur jusqu'à la disparition de ces facultés en 1885, mais, en pratique, le nombre de docteurs en théologie étant insuffisant, il fallut admettre des candidats ne possédant pas ce grade. Le statut des professeurs était, en principe, fixé par l'État, et il en était de même des matières enseignées. Théoriquement, chaque faculté comptait au moins trois professeurs qui dispensaient des cours d'histoire ecclésiastique, de dogme et de morale évangélique.

Fixant, en 1808, le cadre des institutions juives et l'organisation du Consistoire central, Napoléon se préoccupe également que ce troisième culte reconnu puisse dispenser un enseignement théologique de haut niveau. La ville de Metz bénéficiait alors de la belle réputation de sa traditionnelle école talmudique. Celle-ci continua ${ }^{16}$. Afin d'avoir une organisation nationale et une collation des grades correspondant à des critères scientifiques, l'École centrale rabbinique fut ouverte en 1829 à Metz (arrêté du 28 août) par transformation de l'ancienne école talmudique, fort réputée. Elle délivrait, elle aussi, des diplômes nationaux. À partir de 1831, elle fut financée sur fonds publics, tout comme les rabbins furent salariés de l'État. L'école n'accueillait que des étudiants d'un niveau d'étude déjà avancé, au moins bacheliers, et dispensait un enseignement associant les matières religieuses et profanes, dans un esprit moderne et éclairé, comparable à la plupart des mouvements qui se répandaient alors chez les intellectuels juifs en Europe. Il convenait de former les chefs spirituels des temps nouveaux tout au long

15. La faculté de théologie de Toulouse ne fonctionna plus, dès la monarchie de Juillet, car l'archevêque, Mgr D'Astros, refusa de présenter des candidats aux chaires professorales. L'archevêque de Lyon fit de même pendant une douzaine d'années.

16. N. Netter, Metz et son grand passé. Vingt siècles d'Histoire d'une communauté juive, Paris, Jeanne Lafitte, 1999; J. BAUER, L'École rabbinique de France, 1830-1930, Paris, PUF, 1931; R. BERG, Histoire du rabbinat français (XVI $-X^{e} X^{e}$ siècle), Paris, Cerf, 1992. 
d'un cursus s'étalant sur six années, l'étudiant devant passer un examen à la fin de chaque année.

Par décret du 11 juillet 1859, cette école rabbinique de Metz fut transférée à Paris où la communauté juive était nombreuse; elle devint le Séminaire israélite de France, tenant lieu de faculté de théologie; le règlement de l'école était soumis à l'approbation du ministre chargé des cultes (art. 3). Le transfert avait aussi pour objectif de contrecarrer des courants estimés trop traditionalistes qui se développaient à Metz. Isaac Lion Trenel, directeur de l'école de Metz, prit la direction de ce nouvel établissement, charge qu'il assura jusqu'en 1890. Le Séminaire était dirigé par le consistoire de Paris; il comptait environ cinquante élèves. Plusieurs hautes personnalités y enseignèrent, comme Hartwig Derenbourg, membre de l'Institut de France, ou Joseph Halévy. Son financement public cessa en 1905.

\subsection{AVANTAGES ET FAIBLESSES}

Comme toutes les constructions administratives napoléoniennes, l'édification des facultés de théologie correspondit à un souci d'ordre et d'efficacité. L'organisation résistera à la chute de l'empire et fonctionnera au cours de la majeure partie du Xix ${ }^{e}$ siècle. Ces facultés devaient pouvoir répondre aux besoins de la science théologique. Les trois cultes reconnus possédaient leurs établissements. Les facultés étaient assez nombreuses pour le clergé et les fidèles catholiques qui, comme l'avait indiqué le concordat, constituaient la grande majorité des citoyens français; elles respectaient la diversité des deux tendances de la religion protestante et le culte juif n'était pas oublié.

Pourtant, tout au long du siècle, deux insuffisances furent constamment pointées du doigt.

D'une part, ces établissements, catholiques, protestants ou juifs se heurtèrent à une première difficulté, celle de parvenir à réunir un nombre suffisant tant d'étudiants que de professeurs, possédant, les uns et les autres, le niveau d'étude requis. Le Séminaire israélite de France trouva généralement son public. Les facultés de théologie protestante furent parfois dans une situation plus délicate; toutefois, ces établissements demeurant peu nombreux, ceux qui existaient fonctionnèrent de façon assez satisfaisante. La théologie catholique eut plus fréquemment à souffrir d'un manque de professeurs et d'étudiants. Les effectifs restèrent faibles, souvent trop faibles pour que la faculté puisse s'enorgueillir de dispenser un enseignement de haut niveau et de conférer des grades au prestige incontesté. Les étudiants manquaient, 
car les évêques se méfiaient de cet enseignement public dont ils estimaient ne pas contrôler suffisamment le contenu. En outre, ce même épiscopat préférerait des prêtres soumis plutôt que savants. Peu d'étudiants et, parallèlement, difficultés de recrutement des professeurs. Il fut exceptionnel que les jurys de concours de recrutement soient composés de professeurs eux-mêmes docteurs. Il arrivait aussi que l'archevêque ne présente aucun candidat à la nomination. "On s'aperçoit - dit Bruno Neveu - que le corps enseignant des facultés de théologie fut toujours constitué pour une grande part de chargés de cours et de suppléants, dont le statut n'avait pas été fixé par les textes réglementaires $[\ldots]^{17}$ » et, ajoute-t-il, qui n'étaient pas docteurs. Les statuts de chaque faculté prévoyaient la présence d'au moins trois professeurs, recrutés parmi les docteurs. On dut bien souvent se contenter de licenciés ou de chargés de cours aux titres universitaires incertains qui dispensaient la plus grande part des enseignements. Les facultés de théologie catholique furent les plus atteintes par le problème lié aux effectifs. Vers 1870, seule la faculté de théologie de la Sorbonne, à Paris, garde un minimum de notoriété.

Une autre difficulté réelle exista qui, elle aussi, entacha l'autorité dont pouvaient bénéficier les facultés de théologie catholique, sans porter préjudice aux deux autres catégories d'établissements: la construction napoléonienne était l'œuvre unilatérale de l'État, réalisée sans concertation avec Rome. Certes, les évêques ou plus souvent les archevêques gardaient un rôle essentiel puisqu'ils présentaient les candidats aux chaires d'enseignement ${ }^{18}$. Mais le pape n'avait été ni associé aux projets ni invité à approuver leur mise en œuvre. Le service public de l'Université napoléonienne tenait compte des cultes reconnus. Mais il les prenait en considération selon la nature que Napoléon leur avait demandé d'épouser: les cultes étaient, eux aussi, des services publics fonctionnant à l'intérieur du territoire national, à la disposition de l'État. Jamais les diplômes conférés par les facultés de théologie catholique du XIX siècle ne reçurent la consécration d'une reconnaissance canonique de la part du Saint-Siège. Dès le XIX siècle, selon une opinion largement répandue, on considérait que telle était la raison première de l'insuffisance du nombre de prêtres fréquentant ces facultés. Les pouvoirs publics n'y furent pas insensibles et tentèrent quelques aménagements susceptibles de rendre ces facultés plus attractives. L'enseignement dispensé se

17. B. Neveu, op. cit., p. 97.

18. Ce rôle fut d'autant plus important que le concours ne put presque jamais se dérouler régulièrement en raison du trop petit nombre de professeurs docteurs pouvant faire partie du jury. Il en résulta que, dans les faits, l'archevêque bénéficiait d'une très grande liberté de présentation du futur professeur. 
diversifia; au cours de dogme, de morale et d'histoire ecclésiastique s'ajoutèrent ceux de droit canonique, d'Écriture sainte, de langue hébraïque et, à Paris, d'éloquence sacrée. On réaffirma la règle selon laquelle les professeurs étaient certes nommés par le Gouvernement, mais uniquement sur proposition de l'archevêque du lieu. Ces mesures n'eurent que peu de portée; en 1849, certains proposèrent la suppression des facultés de théologie. À cette date, sous le ministère du comte Falloux favorable à l'enseignement libre, une commission présidée par Jean Reynaud envisagea la création de trois grandes écoles de théologie, à Paris, Lyon et Toulouse. Chacune aurait eu sa circonscription territoriale; les évêques et archevêques du lieu auraient formé le haut conseil de l'école, compétent pour approuver les programmes, pour nommer, suspendre ou révoquer les professeurs. L'autorité civile était dépossédée au profit de l'épiscopat. Les évènements politiques et l'arrivée de Fortoul au ministère mirent fin au projet. Le décret-loi du 9 mars 1852, par lequel Fortoul se félicitait « d'avoir sauvé l'Université», pris pour contrecarrer la philosophie des années 1849-1850, attribue de nouveau au président de la République et au ministre une autorité quasi totale sur le corps professoral de l'Université, y compris dans les facultés de théologie.

Le Second Empire avait perçu l'importance de l'enseignement supérieur, mais n'avait pas profondément modifié le système ${ }^{19}$. En 1868, le ministre de l'Instruction publique Victor Duruy instaure l'École pratique des hautes études, une création originale, empreinte d'un grand libéralisme: aucune condition n'est requise pour l'inscription et les directeurs d'étude sont recrutés par cooptation. L'idée était de renouveler les recherches faites dans un cadre universitaire quelque peu sclérosé.

En ce qui concerne les facultés de théologie, la France comptait au début du Second Empire dix-huit églises métropolitaines; en application des textes relatifs à l'Université, il pouvait y avoir autant de facultés de théologie. Pourtant, il n'en exista que six: Bordeaux, Lyon, Aix, Toulouse, Paris et Rouen. À leur propos, un important combat pour une reconnaissance canonique des diplômes délivrés fut mené par les plus hauts membres de l'épiscopat ou de ces facultés. On faisait valoir qu'une université dépourvue de reconnaissance expresse de ses diplômes par le Saint-Siège ne devait pas pouvoir se prévaloir du qualificatif de catholique; cette absence d'homologation romaine discréditait effectivement l'institution auprès de l'épiscopat et plus largement du monde catholique en France. Ce fut en vain que Mgr Maret, doyen de la

19. J. Maurain, La politique ecclésiastique du Second Empire de 1852 à 1869, Paris, Félix Alcan, 1930 , spéc. p. 104 , p. 146 , p. 210 , p. 580 , p. 748 , p. 855. 
faculté de la Sorbonne, intervint activement en ce sens, à Paris et à Rome ${ }^{20}$; bien qu'il ait bénéficié de l'appui du ministre Fortoul, Mgr Maret n'obtint pas gain de cause, en raison des tensions qui commençaient à se développer entre l'empereur et le pape.

\section{LES FACULTÉS DE THÉOLOGIE SOUS LA IIIE RÉPUBLIQUE, ENTENTE, DIVORCE ET RÉUSSITE LOCALE}

À partir des années 1870, l'histoire des facultés de théologie connut trois grands bouleversements, liés les uns et les autres aux évènements politiques touchant l'ensemble de la France. C'est dire que ces établissements constituent - ou constituaient - un défi de premier ordre dans le jeu des forces politiques et notamment dans les oscillations entre cléricalisme et anticléricalisme des gouvernements successifs.

\subsection{LA CRÉATION D'UNIVERSITÉS LIBRES}

En 1873, lors de la période dite de l'Ordre moral, la France est officiellement en République, mais une forte majorité de royalistes détiennent tous les postes clés; les députés royalistes entendent profiter de cette situation pour rétablir une royauté en France. Ils voient dans l'Église une force susceptible de leur apporter des appuis considérables. Mgr Maret, toujours doyen de la faculté de théologie de l'université de Paris, reprend son combat pour la reconnaissance canonique des diplômes conférés par la faculté dont il est en charge, à la Sorbonne. Son collègue dans le décanat de la faculté de Lyon le rejoint dans cette démarche ${ }^{21}$. Tous deux déplorent le peu d'intérêt que des ecclésiastiques peuvent trouver dans des grades de théologie reconnus par l'État mais non par l'Église.

Au cours de cette même période, les Chambres se montrent très sensibles aux demandes de l'Église. Dans ce contexte est votée la loi du 12 juillet $1875^{22}$

20. Mgr Maret fut nommé professeur de dogme à la faculté de théologie de la Sorbonne en 1841 et devint doyen en 1853; V. notamment: C. BRESSOLETTE (éd.), Henry Maret, L'Église et l'État: cours de Sorbonne inédit (1850-1851), Paris, Beauchesne, 1979.

21. B. Neveu, op. cit., p. 408; C. Bressolette, «Les derniers combats de Mgr Maret pour le maintien de la faculté de théologie de Sorbonne», in Humanisme et foi chrétienne. Mélanges scientifiques du centenaire de l'Institut catholique de Paris, Paris, Beauchesne, 1976, p. 49-69.

22. P.-H. PRÉLOT, Naissance de l'enseignement supérieur libre: la loi du 12 juillet 1875, Paris, PUF, 1987; Les établissements privés d'enseignement supérieur, Paris, LGDJ, 1989 ; B. NeVEU, 
autorisant la création d'établissements d'enseignement supérieur libres, qui pourront s'appeler facultés dès lors qu'ils réuniront un nombre de professeurs docteurs au moins équivalent à celui des plus petites facultés d'État. Trois facultés libres d'une même ville peuvent former une université libre. Les étudiants passeront leurs examens soit devant un jury de faculté d'État, soit devant un jury mixte composé pour moitié de professeurs d'une faculté d'État et pour moitié de professeurs d'une faculté libre. Les diplômes demeurent des diplômes d'État. Pour les catholiques de France, l'enjeu est considérable et c'est par eux que la loi va être utilisée. Celle-ci fut, dans son ensemble, appréciée par les catholiques qui créèrent des universités catholiques ${ }^{23}$. Mais si le vote était intervenu dans un climat politique favorable à l'Église, la mise en ouvre se situera rapidement dans un tout autre contexte.

En outre, la loi plaçait l'enseignement de la théologie dans une situation délicate ${ }^{24}$ : fallait-il créer des facultés libres de théologie? La loi en donnait la possibilité. Celles existant alors dans le cadre de l'Université de France, université publique, n'avaient qu'un faible rayonnement et surtout peu d'étudiants. Pourraient-elles subsister si d'autres, bénéficiant d'une reconnaissance canonique, leur faisaient concurrence? Mgr Maret et bien d'autres avaient déjà exprimé leurs craintes et leur désir d'une autre solution, celle consistant à faire reconnaître par le Saint-Siège valeur canonique aux diplômes délivrés dans le cadre des facultés de théologie de l'Université. Par ailleurs, des facultés de théologie au sein d'universités catholiques risquaient de dispenser des doctrines ultramontaines virulentes qui allaient ruiner tout effort de rapprochement entre Rome et la République. L'épiscopat était loin d'apprécier sans réserve la création de facultés de théologie conformes à la loi de 1875 . Outre les rivalités qui naîtraient inévitablement entre établissements publics

«L'enseignement universitaire de la théologie catholique en France de 1875 à 1885 », in G. Cholvy et N.-J. Chaline (dir.), L'enseignement catholique en France aux XIX et XXe siècles, Paris, Cerf, 1995, p. 269-294, également publié dans RHEF, no 206, 1995, p. 269-294: https://www.persee.fr/doc/rhef_0300-9505_1995_num_81_206_1187 [consulté le 4 janv. 2020].

23. A. Baudrillart, «La fondation de l'université catholique de Paris en 1975 », Revue d'histoire de l'Église de France, 1912, p. 1-37; C. NASTORG, «L'Institut catholique de Toulouse. Les années de fondation", in G. Cholvy et N.-J. Chaline (dir.), op. cit., p. 255-268; M. BISCAY, «L'esprit de la faculté catholique de droit de Lyon de 1875 à 1880: faculté libre ou contre-faculté?», Revue d'histoire des facultés de droit et de la culture juridique, $\mathrm{n}^{\circ} 37,2017$, p. 165-182.

24. B. BASDEVANT-GAudEMET, « Diritto ecclesiastico ou droit des cultes, une discipline universitaire en Italie et en France aux XIX ${ }^{\mathrm{e}}-\mathrm{XX}^{\mathrm{e}}$ siècles», in M. CAvina (a cura di), L'insegnamento del diritto (secoli XII-XX) = L'enseignement du droit (XII $-X X^{e}$ siècles), Bologna, Il Mulino, 2019, p. 363-382. 
et privés, les évêques déploraient que cette législation maintienne une emprise étatique sur la collation des grades puisque les examens se passaient devant des jurys mixtes, même pour la théologie.

En définitive, entre 1875 et 1879, cinq universités libres ouvrirent néanmoins un enseignement de théologie catholique, dans des établissements qualifiés d'école supérieure, de collège, ou de faculté ${ }^{25}$. La création de ces cursus avait suscité bien des débats; entre les évêques, l'autorité romaine, l'Université publique, le Gouvernement, les discussions furent âpres, chacun se méfiant de l'orientation de la doctrine qui allait être dispensée par le nouvel établissement. Finalement, les facultés de théologie pouvaient exister dans l'Université de France, et d'autres pouvaient être érigées en application de la loi de 1875. La concurrence ne fut propice au rayonnement d'aucune des deux catégories ${ }^{26}$. Les facultés de théologie de l'Université de France connaissaient toujours les mêmes difficultés de fonctionnement: effectifs faibles et enseignants ne pouvant prétendre au titre de professeur ${ }^{27}$. Quant au fonctionnement des universités libres de la loi de 1875, il ne fut que de courte durée.

Par ailleurs, si les premières années de la Troisième République furent favorables à l'Église catholique, le régime de liberté religieuse et l'existence des quatre cultes reconnus conduisirent naturellement au maintien du séminaire juif et, nous l'avons dit, à la création de la faculté de théologie protestante de Paris en 1877 dont les débuts ont été fort bien analysés par André Encrevée 28 .

25. Il y eut: une faculté de théologie à Angers puis à Paris, une école supérieure de théologie à Lyon et à Toulouse, un collège théologique à Lille. Sur le fonctionnement, difficile, de ces établissements, V. B. NEvEU, op. cit.: l'auteur détaille magnifiquement la vie de ces écoles ou facultés; il décrit aussi l'intéressante tentative de Mgr Pie, à Poitiers. Mgr Pie, évêque de Poitiers, profondément ultramontain et de forte personnalité, avait créé, avec l'accord de Rome, une faculté de théologie qui n'entrait nullement dans le cadre de la loi de 1875; il s'était abstenu de soumettre le bref d'érection de l'établissement à réception par le Conseil d'État et les évêques de sa province n'avaient pas donné leur assentiment; les enseignants venaient essentiellement du collège romain; cette faculté disparut à la mort de Mgr Pie en 1880.

26. B. NeVEu, «L'enseignement universitaire de la théologie catholique en France de 1875 à $1885 »$, op. cit.

27. J.-O. Boudon, «L'épiscopat français et le développement des hautes études ecclésiastiques au XIX ${ }^{e}$ siècle», Revue d'histoire de l'Église de France, no 206, 1995, p. 219-235; l'auteur expose plusieurs intéressantes tentatives épiscopales menées afin de rehausser tant le niveau scientifique que l'attrait de ces études; tentatives sans résultats véritablement positifs.

28. A. ENCREVÉ, «La fondation et les premières années de la faculté de théologie protestante de Paris (1877-1882)», Études théologiques et religieuses, 2011/3, p. 321-333. 


\subsection{LA SUPPRESSION DES FACULTÉS DE THÉOLOGIE DE L'UNIVERSITÉ PUBLIQUE}

Les élections de 1876 avaient permis aux républicains d'obtenir une confortable majorité à la Chambre des députés; après la crise du 16 mai 1877, le régime républicain avait consolidé son accession au pouvoir. Les universités libres mises en place par la hiérarchie catholique firent rapidement les frais de ce changement de majorité. La loi du 18 mars 1880 supprime les jurys mixtes; désormais, seuls des professeurs de l'Université de France sont habilités à siéger dans des jurys conférant les grades universitaires: baccalauréat, licence, doctorat. Les établissements privés n'ont plus le droit de se qualifier d'universités ni d'accorder ces grades ${ }^{29}$; ils deviennent des instituts catholiques. Le nombre d'étudiants, déjà faible, décline encore nettement. La loi de finances du 21 mars 1885 supprimait les crédits jusqu'alors alloués aux facultés de théologie catholiques de l'Université de France, ce qui entraîna, dès la rentrée suivante, la disparition de ces facultés. Une loi du 27 juin 1885 accordait aux anciens professeurs une allocation annuelle sur les crédits de l'Instruction publique. En France, les facultés de théologie organisées au sein de l'Université publique avaient vécu. Telle est l'histoire spécifique à la France, étroitement liée aux vicissitudes des régimes politiques et qui ne se retrouve pas dans les autres pays européens. La concurrence qui avait opposé les deux catégories de facultés de théologie pendant dix ans n'avait profité ni aux unes ni aux autres. L'enseignement de la théologie catholique semblait dans une situation critique. À l'initiative de Louis Liard, alors directeur de l'Enseignement supérieur, un décret du 30 janvier1886 instaurait à l'École pratique des hautes études, comme une sorte de compensation, une cinquième section, la section des sciences religieuses ${ }^{30}$. Le décret spécifiait qu'il s'agissait d'étudier les textes, non les dogmes; on s'inscrivait dans ce mouvement qui reconnaît la place des sciences religieuses comme aidant à

29. Sur la question fondamentale du monopole de la collation des grades, V. les écrits d'Yves Gaudemet et ceux de Pierre-Henri Prélot; les deux éminents professeurs de droit public défendent des opinions différentes dans un même volume; trois articles dans: Les facultés de droit demain?, numéro spécial de la RDP 2013: Y. GAUDEMET, «Le monopole de la collation des grades universitaires: quelques remarques introductives à la lecture de l'article de Pierre-Henri Prélot et sur son invitation», p. 281-287; P.-H. PrÉLOT, «Le monopole de la collation des grades. Étude historique du droit positif et de ses évolutions contemporaines», p. 287-326; Y. GAUDEMET, « Notes sur la délivrance des grades et diplômes nationaux et spécialement du master et sur les établissements publics de l'État pouvant être habilités à délivrer des diplômes nationaux », p. 327-331. Les deux premiers de ces trois articles sont des reprises d'articles publiés dans cette même revue en 2008.

30. M. Vernes, «Les caractéristiques de l'École pratique des Hautes Études - Sciences religieuses et sa place dans l'enseignement supérieur français», in École pratique des hautes études, Section des sciences religieuses. Annuaire, no 25, 1915, p. 19-45. 
la connaissance des sociétés, mais en passant du domaine du dogme à celui de l'histoire. Les facultés de théologie protestante, qui servaient également de séminaires pour les pasteurs, subsistèrent et ne seront supprimées qu'en application de la loi de séparation des Églises et de l'État du 9 décembre 1905.

Le temps des facultés de théologie au sein de l'Université publique était alors révolu en France, pour toutes les confessions. Bien des écoles ou instituts se créèrent, qui fonctionnèrent et fonctionnent. Beaucoup obtiennent d'importantes subventions publiques et jouissent d'une réelle notoriété. Pourtant, le recrutement du corps professoral ne se fait pas selon les mêmes procédures qu'au sein des universités et les grades acquis ne sont pas des diplômes conférés par l'État. Cette actualité dépasse le cadre historique de cette contribution.

Une exception essentielle et particulièrement prestigieuse existe néanmoins à Strasbourg.

\subsection{LE CAS DE STRASBOURG}

Les facultés de théologie, catholique et protestante, qui existent au sein de l'université publique de Strasbourg sont, elles aussi, le fruit de l'histoire $^{31}$. La ville de Strasbourg s'était trouvée rattachée à l'Allemagne suite à la défaite de 1870. La faculté de théologie protestante est intégrée à la nouvelle université allemande dès sa création par la loi du 28 avril $1872^{32}$. Des négociations commencèrent, à cette date, en vue d'une réorganisation de l'enseignement théologique catholique au sein de l'université à côté de la faculté de théologie protestante, toujours active. L'évêque de Strasbourg est hésitant, craignant une germanisation du clergé. Ce fut seulement le 5 décembre 1902 qu'intervint un accord portant érection d'une faculté de théologie catholique à l'université de Strasbourg. La convention fut signée entre le cardinal Rampolla, secrétaire d'État du pape Léon XIII, et le baron Georges de Hertling, professeur à l'université de Munich et représentant du gouvernement impérial allemand. Cette faculté était organisée sur le modèle

31. B. Le LéanneC, «L'enseignement supérieur», in J. SChlick et M. Zimmermann (dir.), Églises et État en Alsace Moselle, Strasbourg, Cerdic, 1979, p. 301-316; FACUltÉ DE THÉOLOGIE CAtholique, Mémorial du cinquantenaire: 1919-1969, Strasbourg, Palais universitaire, 1970, V. notamment l'article de C. Vogel, «La faculté de théologie catholique de 1902 à 1918 », p. 37-75; F. MESSNER, «Formation des ministres du culte...», art. cit.

32. R. Minnerath, «Le concordat de Bonaparte et son actualité», Napoleonica. La Revue, 2015/2, p. 4-20: https://www.cairn.info/revue-napoleonica-la-revue-2015-2-page-4. htm [consulté le 4 janv. 2020]. 
de celles, prestigieuses, de Bonn et de Breslau. En octobre 1903, la faculté de théologie catholique fut effectivement ouverte, à côté de celle relative au protestantisme. Par le bref Venerabilis du 3 septembre 1903, Pie X lui avait accordé le droit de conférer les grades canoniques de licencié et de docteur. L'évêque de Strasbourg et le grand séminaire furent associés à l'élaboration des statuts et divers règlements précisant le mode de fonctionnement de cette faculté.

Cette création allemande s'intégrait harmonieusement dans le système universitaire allemand. Comment put-elle subsister après $1918^{33}$ ? Réorganisant l'université de Strasbourg ${ }^{34}$, le gouvernement français fut soucieux de doter cette université de tous les moyens lui permettant d'acquérir un prestige exceptionnel témoignant de son retour à la France. Les facultés de théologie en bénéficièrent tout particulièrement et furent à l'honneur le jour de la rentrée solennelle de l'Université française, le 22 novembre 1919. Dans une allocution du 21 novembre 1921, le pape Benoît XV disait qu'il considérait comme caducs les accords signés avec des entités dont les traités de paix avaient substantiellement modifié l'identité. La France demanda donc expressément que la convention de 1902 soit maintenue. Un nouvel accord fut signé en ce sens entre Raymond Poincaré, président du Conseil et le cardinal Gasparri, secrétaire d'État. Le Saint-Siège reconnaissait le caractère canonique de la faculté et des grades décernés. La France s'engageait à ne nommer un professeur qu'après avis de l'archevêque de Strasbourg. Ces tractations aboutirent au décret du président de la République du 30 mai 1924 plaçant une faculté de théologie catholique reconnue par l'autorité romaine et une faculté de théologie protestante, toutes deux à l'université publique de Strasbourg.

Si, à Strasbourg, l'Église catholique connaît toujours le régime concordataire napoléonien, notons cependant que ce n'est pas en raison du maintien du concordat que l'université de Strasbourg délivre des diplômes reconnus tout à la fois par l'Église et par l'État. D'ailleurs, une telle réussite n'avait jamais été possible dans les autres universités françaises, au XIX siècle, lorsque le régime concordataire était en vigueur. La condition de Strasbourg est directement liée à la convention de 1902, convention dont les termes semblaient

33. Ch. Haubert, «La faculté française de théologie catholique (1919)», in FAculté DE THÉoLOGIE CATHOLIQUE, op. cit., p. 76-80; l'histoire des années et décennies suivantes de cette faculté est décrite dans les autres articles de ce volume.

34. J. Gaudemet, «Les débuts de la Faculté de droit et des sciences politiques de Strasbourg au lendemain de la première guerre mondiale (novembre 1918-novembre 1919)», Revue d'histoire des facultés de droit et de la culture juridique, $\mathrm{n}^{\circ}$ 18, 1997, p. 95-124. 
assez ordinaires pour les universitaires ou les pouvoirs publics allemands, contrairement à la France.

Mise à part la merveilleuse réussite spécifique de l'université strasbourgeoise, il faut bien admettre que la période de grandeur des facultés de théologie au sein des universités françaises remonte, pour le moins, à l'Ancien Régime et, diraient certains, au Moyen Âge. Il serait simpliste de voir ici la marque de l'anticléricalisme ou de la laïcité à la française. Sans doute ces facteurs ont-ils joué, mais il importe de les replacer au milieu d'une histoire plus générale de l'État, des régimes politiques, de l'enseignement et des religions en France. L'autoritarisme napoléonien avait instauré le monopole du service public de l'Université impériale dans un esprit gallican qui ne se voulait pas anticlérical, mais qui fut anti-romain. Dès lors, le recrutement des professeurs et la collation des grades ne pouvaient relever que de l'État, monopole qui constitue un utile garant du niveau scientifique des universités. Mais le moule était-il adapté aux facultés de théologie catholique? Dans l'esprit de Napoléon, il ne devait pas heurter l'Église, car celle-ci était intégrée à la construction d'ensemble. Pourtant, le gallicanisme des gouvernements du XIXe siècle ne fit pas toujours bon ménage avec l'épiscopat. Les initiatives de l'État n'ont jamais reçu de consécration romaine. Ailleurs, la convention dont bénéficia Strasbourg procédait, dès son origine, d'autres sources d'inspiration. 